\title{
Aqueous Oldenlandia diffusa extracts inhibits colorectal cancer cells via activating AMP-activated protein kinase signalings
}

\author{
Pei-Hua Lu' ${ }^{1,2, *}$, Min-Bin Chen ${ }^{3, *}$, Chao $\mathrm{Ji}^{4,}{ }^{*}$, Wen-Ting $\mathrm{Li}^{1}$, Mu-Xin Wei ${ }^{5}$, Mian-Hua Wu ${ }^{1}$ \\ ${ }^{1}$ Jiangsu Collaborative Innovation Center of Traditional Chinese Medicine (TCM) Prevention and Treatment of Tumor, Nanjing \\ University of Chinese Medicine, Nanjing, 210023, China \\ ${ }^{2}$ Department of Medical Oncology, Wuxi People's Hospital Affiliated to Nanjing Medical University, Wuxi, 214023, Jiangsu, \\ China \\ ${ }^{3}$ Department of Medical Oncology, Kunshan First People's Hospital Affiliated to Jiangsu University, Kunshan, 215300, Jiangsu, \\ China \\ ${ }^{4}$ Department of Dermatology, The First Affiliated Hospital of Fujian Medical University, Fuzhou, 350005, Fujian, China \\ ${ }^{5}$ Department of Traditional Chinese Medicine, First Affiliated Hospital of Nanjing Medical University, Nanjing, 210029, China \\ *These authors have contributed equally to this work \\ Correspondence to: Mian-Hua Wu, email: duoduoli772@sina.com \\ Keywords: colorectal cancer (CRC), Oldenlandia diffusa (OD) extracts (ODE), AMP-activated protein kinase (AMPK), p53 and \\ mammalian target of rapamycin (mTOR) \\ Received: April 18,2016 Accepted: June 03, $2016 \quad$ Published: June 13, 2016
}

\section{ABSTRACT}

Here we evaluated the anti-cancer activity of aqueous Oldenlandia diffusa (OD) extracts (ODE) in colorectal cancer (CRC) cells. We showed that ODE exerted potent anti-proliferative, cytotoxic and pro-apoptotic activities against a panel of established CRC lines (HCT-116, DLD-1, HT-29 and Lovo) and primary (patient-derived) human CRC cells. ODE activated AMP-activated protein kinase (AMPK) signaling, which led to subsequent mTORC1 inhibition and Bcl-2/HIF-1 $\alpha$ downregulation in CRC cells. In ODE-treated CRC cells, AMPK $\alpha 1$ formed a complex with p53. This might be important for p53 activation and subsequent cancer cell apoptosis. Inhibition of AMPK signaling, though dominant negative (dn) mutation or shRNA/siRNA knockdown of AMPK $\alpha 1$ attenuated ODE-exerted CRC cytotoxicity. In vivo, i.p. administration of ODE inhibited HCT-116 xenograft tumor growth in SCID mice. In addition, AMPK activation, mTORC1 inhibition and p53 activation were observed in ODE-treated HCT-116 xenograft tumors. These results suggest that ODE inhibits CRC cells in vitro and in vivo, possibly via activation of AMPK-dependent signalings.

\section{INTRODUCTION}

The application of conventional chemotherapy is limited in colorectal cancer (CRC) cells with pre-existing and/or acquired resistances [1]. In addition, our groups [2-4] and others have shown that molecular heterogeneity of CRCs hinders the uniform application of specific molecularly-targeted agents [5-7]. Therefore, studies are exploring novel and more efficient anti-CRC agents [8].

Oldenlandia diffusa (OD), a member of the Rubiaceae family, is a well-known medicinal plant in ancient China [9]. Existing evidences have described multiple biological functions of OD components, including anti-angiogenic, anti-inflammatory, anti-oxidant, and pro-apoptotic activities $[9,10]$. More importantly, (OD) extracts (ODE) have displayed significant anticancer activity in a number of preclinical cancer studies [10-13]. However, the potential effect of ODE in CRC cells has not been extensively studied.

Our studies [14, 15] have implied that AMPactivated protein kinase (AMPK), the master energy sensor, is also an important mediator of cell death and apoptosis under various stress conditions (see review [16]). In multiple cancer cell lines, various anti-cancer agents and natural occurring compounds were shown to activate AMPK-dependent cell apoptosis/death pathways 
$[14,16-26]$. In the current study, we show that ODE potently inhibits CRC cells in vitro and in vivo. Activation of AMPK could be the major signaling mechanisms responsible for ODE's actions in CRC cells.

\section{RESULTS}

\section{Oldenlandia diffusa extracts (ODE) inhibits CRC cell proliferation and survival}

MTT assay results in Figure 1A showed that ODE inhibited HCT-116 cell proliferation (MTT viability reduction). The anti-proliferative activity by ODE in HCT116 cells was concentration- and time-dependent (Figure 1A). The colony formation assay results in Figure $1 \mathrm{~B}$ and BrdU incorporation assay in Figure 1C further confirmed the anti-proliferative activity of ODE when applied in HCT-116 cells. The number of proliferative HCT-116 colonies (Figure 1B) and BrdU incorporation (Figure 1C) were both dramatically decreased after ODE $(25-200 \mu \mathrm{g} /$ $\mathrm{mL})$ treatment. A low-concentration of ODE $(10 \mu \mathrm{g} / \mathrm{mL})$ showed no significant effect on HCT-116 cell proliferation
(Figure 1B and 1C, $p>0.05 v s$. control group). Trypan blue staining assay results in Figure 1D demonstrated that ODE at $25-200 \mu \mathrm{g} / \mathrm{mL}$ induced significant HCT-116 cell death.

Next, we studied the potential activity of ODE to other human CRC cells. MTT results in Figure 1E showed that ODE $(50 \mu \mathrm{g} / \mathrm{mL})$ inhibited the proliferation of three other established CRC cell lines, including DLD-1, HT29 and Lovo. We also calculated the IC-50 of ODE in above CRC cells with different $\mathrm{p} 53$ status. The IC-50 of ODE was low in p53-wild HCT-116 $(33.57 \pm 2.57 \mu \mathrm{g} / \mathrm{mL})$ and LoVo $(12.33 \pm 1.51 \mu \mathrm{g} / \mathrm{mL})$ CRC cells [33-35], but was relatively high in p53-mutant HT-29 $(55.56 \pm 3.57 \mu \mathrm{g} /$ $\mathrm{mL})$ and DLD-1 $(42.31 \pm 3.32 \mu \mathrm{g} / \mathrm{mL})$ cells [33-35]. Meanwhile, we established three lines of patient-derived primary CRC cells based on the method described [2]. These primary CRC cells were also incubated with ODEcontaining medium. MTT assay was again performed, and results (Figure $1 \mathrm{~F})$ showed that $\mathrm{ODE}(50 \mu \mathrm{g} / \mathrm{mL})$ inhibited proliferation of all three lines of primary CRC cells. Together, these results show that ODE exerts potent anti-proliferative and cytotoxic activity against human CRC cells.
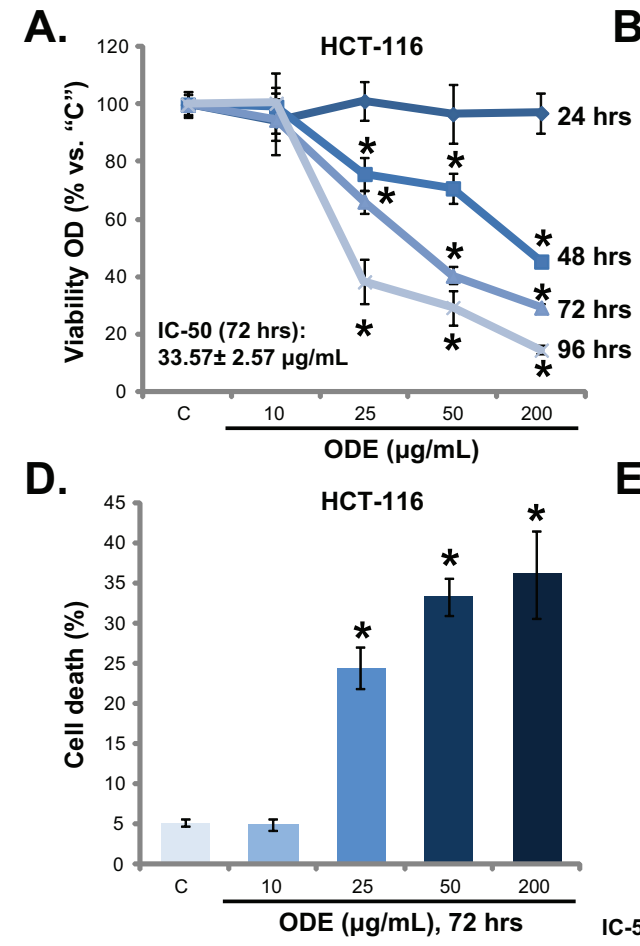

B.

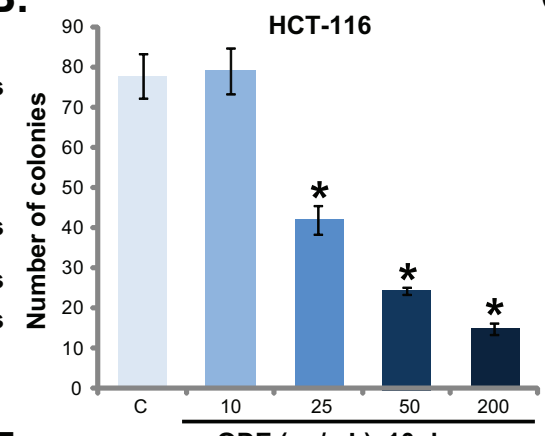

E.

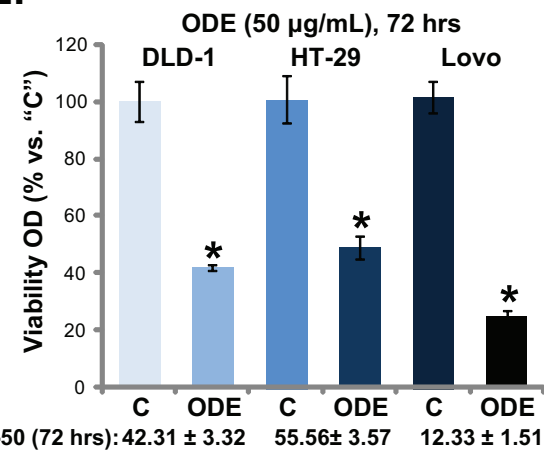

C.
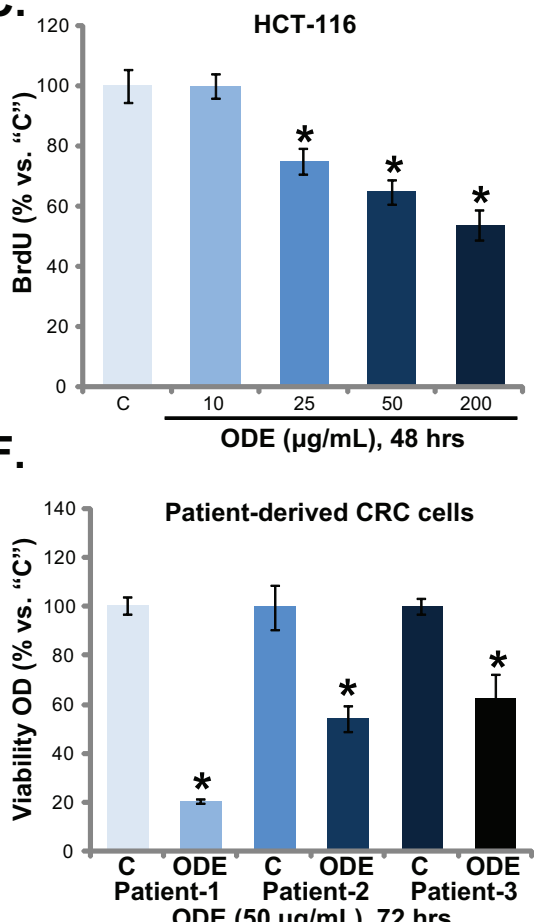

Figure 1: Oldenlandia diffusa extracts (ODE) inhibits CRC cell proliferation and survival. A panel of established CRC cell lines (HCT-116, Lovo, HT-29 and DLD-1) or three primary human CRC cell lines were treated with or without ODE at applied concentrations, cells were further cultured, and cell proliferation was evaluated by MTT assay A, E and $\mathbf{F}$., colony formation assay (B., for HCT-116 cells) and BrdU incorporation assay (C., for HCT-116); Cell death was analyzed by the trypan staining assay (D., for HCT-116). "C" stands for untreated control group (Same for all Figures). For each assay, n=5 (Same for all Figures). Data in this figure were repeated four times, and similar results were obtained. * $p<0.05$ vs. "C" group. 


\section{ODE activates apoptosis in CRC cells}

Next, several apoptosis assays were performed to test cell apoptosis in ODE-treated CRC cells. Results demonstrated that ODE $(25-200 \mu \mathrm{g} / \mathrm{mL})$ induced significant apoptosis activation in HCT-116 cells. The caspase-3 activity (Figure 2A), Histone DNA ELISA OD (Figure 2B), the percentage of Annexin V or TUNEL positive cells (Figure $2 \mathrm{C}$ ) were all increased following ODE $(25-200 \mu \mathrm{g} / \mathrm{mL})$ treatment in HCT-116 cells. Meanwhile, the expressions of cleaved-poly (ADPribose) polymerase (PARP) and cleaved-caspase-3 were increased in ODE (25-200 $\mu \mathrm{g} / \mathrm{mL})$-treated HCT-116 cells (Figure 2D). Once again, the low-concentration of ODE $(10 \mu \mathrm{g} / \mathrm{mL})$ showed no significant effect on HCT-116 cell apoptosis (Figure 2A-2D).
Next, the apoptosis inhibitors, including the caspase-3 specific inhibitor Ac-DEVD-CHO, the caspae-9 specific inhibitor Ac-LEHD-CHO and the caspae-8 specific inhibitor Ac-ITED-CHO, were applied. MTT assay results in Figure 2E and trypan blue staining assay results in Figure 2F showed that the three caspase inhibitors significantly attenuated $\operatorname{ODE}(50 \mu \mathrm{g} / \mathrm{mL})$ induced anti-proliferative and cytotoxic actions against HCT-116 cells. These results suggest that caspasedependent apoptosis activation mediates ODE-induced anti-CRC cell activity. Further, ODE $(50 \mu \mathrm{g} / \mathrm{mL})$ was again pro-apoptotic in other established (DLD-1, HT-29 and Lovo) (Figure 2G) and primary (Figure 2H) human $\mathrm{CRC}$ cells. Note that apoptosis was tested by histone-DNA ELISA assay (Figure $2 \mathrm{G}$ and $2 \mathrm{H}$ ). The above caspase inhibitors similarly alleviated ODE-mediated cytotoxicity
A.

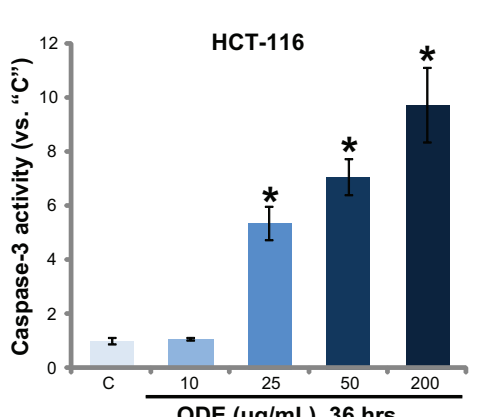

D.

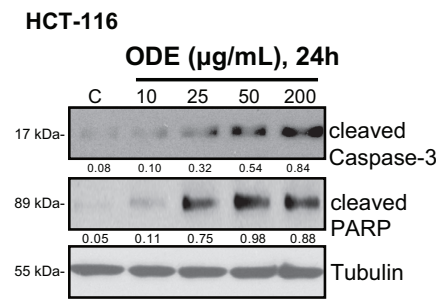

G.

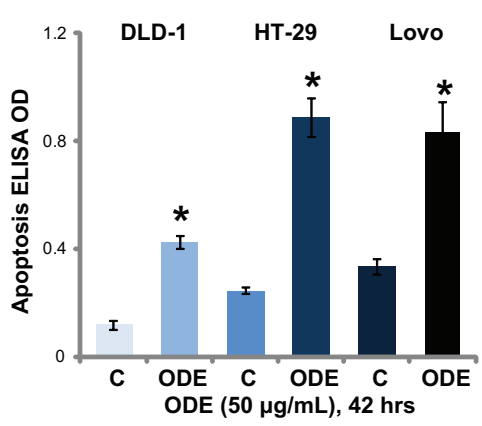

B.

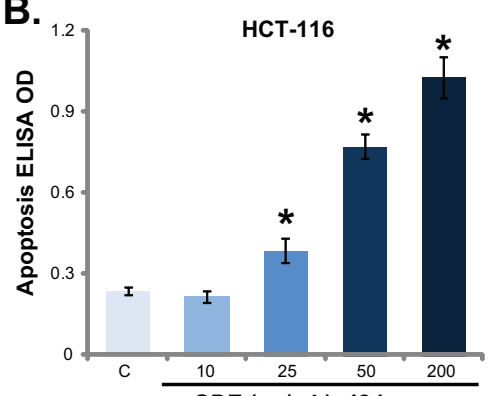

E.

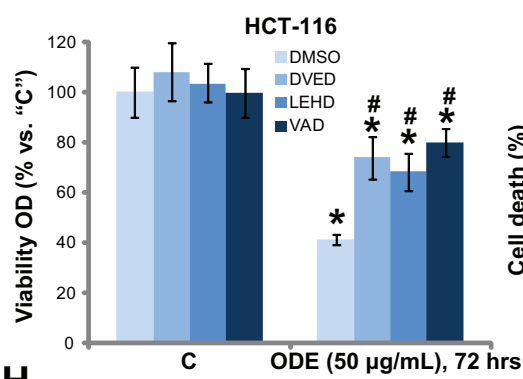

H.

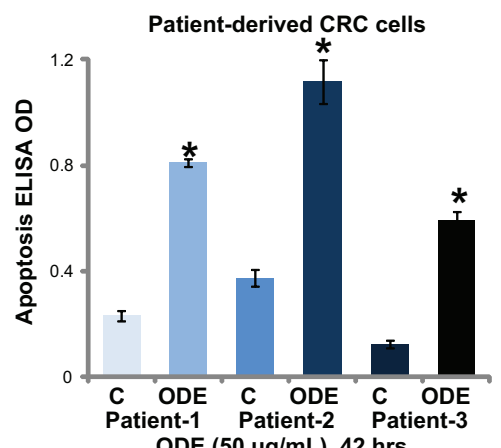

C.

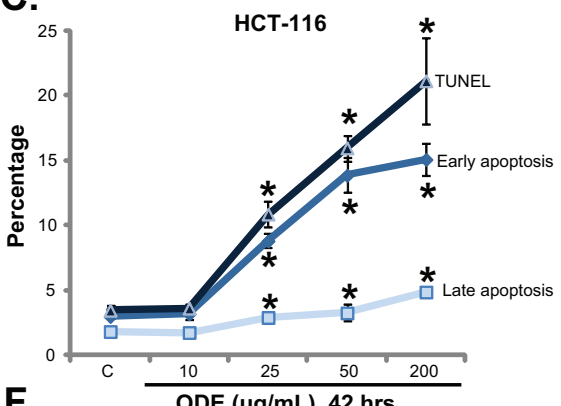

$\mathbf{F}$.

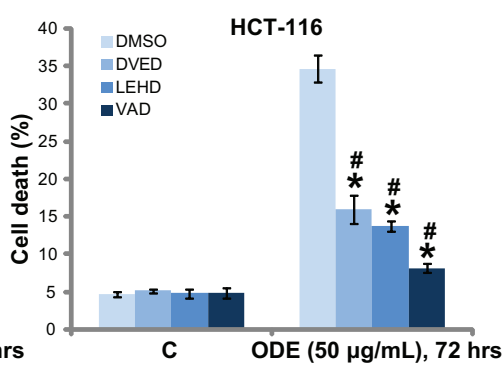

ODE $(50 \mu \mathrm{g} / \mathrm{mL}), 42 \mathrm{hrs}$

Figure 2: ODE activates apoptosis in CRC cells. A panel of established CRC cell lines (HCT-116, Lovo, HT-29 and DLD-1) and three primary human CRC cell lines were treated with or without ODE at applied concentrations, cells were further cultured, cell apoptosis was analyzed by listed assay A-D, G and H. HCT-116 cells were pretreated with Ac-DEVD-CHO ("DVED"), Ac-LEHD-CHO ("LEHD") or Ac-VAD-CHO ("VAD") (40 $\mu \mathrm{M}$ each) for $1 \mathrm{~h}$, following by ODE $(50 \mu \mathrm{g} / \mathrm{mL})$ treatment, cell viability E. and cell death $\mathbf{F}$. were tested. "DMSO" stands for 0.1\% DMSO. Cleaved-PARP/cleaved-caspase-3 expression (vs. Tubulin) was quantified. Data in this figure were repeated four times, and similar results were obtained. ${ }^{*} p<0.05$ vs. "C" group. ${ }^{*} p<0.05$ vs. "ODE" only group (E and F). 
against above established (DLD-1, HT-29 and Lovo) and primary CRC cells (Data not shown). Collectively, these results show that ODE induces apoptotic death in cultured CRC cells.

\section{ODE activates AMPK signaling in CRC cells}

Existing evidences implied that AMPK activation inhibits cancer cells through regulating its downstream signals, including activating pro-apoptotic p53 cascades, and inactivating pro-cancerous mTOR complex 1 (mTORC1) signaling [16]. As shown in Figure 3A, ODE treatment in HCT-116 cells induced significant AMPK signaling activation, evidenced by AMPK $\alpha 1 /$ ACC phosphorylations [29]. Activation of mTORC1 (p-S6K1), and expressions of mTORC1-dependent genes
(Bcl-2 [28, 29] and HIF-1 $\alpha[36,37])$ were remarkably inhibited by ODE treatment in HCT-116 cells (Figure 3B). Inactivation of AMPK, via AMPK $\alpha 1$ shRNA (Figure 3C) [29] or dominant negative (dn) mutation (T172A, Figure 3C) [29], restored S6K1 phosphorylation (Figure 3D) and Bcl-2/HIF-1 $\alpha$ expression in ODE-treated HCT-116 cells (Figure 3D). These results suggest that ODE activates AMPK signaling to inhibit mTOR activation (p-S6K1) and mTOR-regulated gene (Bcl-2 and HIF- $1 \alpha$ ) expression in HCT-116 cells. Similar results were also obtained in HT-29 cells and DLD-1 cells (Data not shown). In primary human CRC cells (patient-1 derived), ODEtreatment also activated AMPK signaling (AMPK $\alpha / \mathrm{ACC}$ phosphorylations) (Figure 3E). p-S6K1 and Bcl-2/HIF-1 $\alpha$ expressions were also inhibited (Figure 3F). Same results were seen in two other primary CRC cell lines (Data not

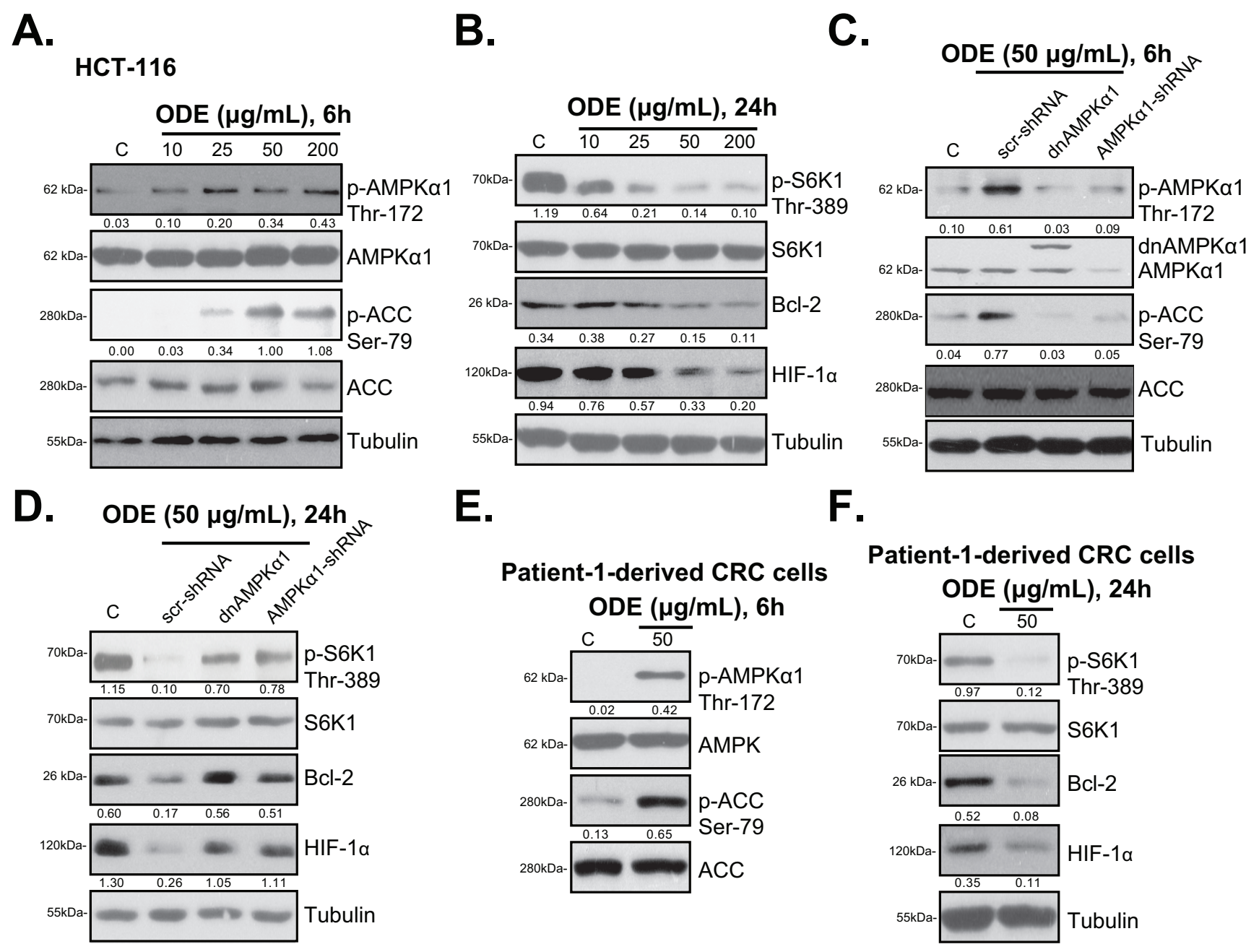

Figure 3: ODE activates AMPK signaling in CRC cells. HCT-116 cells or patient-1-derived primary CRC cells were treated with or without applied ODE, cells were further cultured, expressions of listed proteins were tested by Western blots A, B, E and F. Stable HCT116 cells expressing scramble control shRNA ("scr-shRNA"), AMPK $\alpha 1$-shRNA or dominant negative (dn)-AMPK $\alpha 1$ ("dnAMPK $\alpha 1$ ") were treated with or without applied ODE, cells were further cultured for $6 \mathrm{~h} \mathrm{C}$. or $24 \mathrm{~h} \mathrm{D.}$., expressions of listed proteins were tested by Western blots. Kinase phosphorylations and Bcl-2/HIF-1 $\alpha$ expressions were quantified. Data in this figure were repeated three times, and similar results were obtained. 
shown). Thus, these results suggest that ODE activates AMPK to inhibit mTORC1 activation in CRC cells.

\section{AMPK activation mediates ODE-induced anti- CRC cell activity}

Using the same genetic strategies, we showed that ODE-exerted HCT-116 cell viability reduction (Figure 4A), cell death (Figure 4B) and apoptosis (Figure 4C) were significantly attenuated with AMPK $\alpha 1$ silencing or mutation. Similar results were also obtained in HT-29 cells (Data not shown). Thus, we propose that ODE treatment in CRC cells induces a profound AMPK activation, causing mTORC1 in-activation, Bcl-2/HIF-1 $\alpha$ downregulation, which might be responsible for $\mathrm{CRC}$ cell growth inhibition and apoptosis. In patient (-1)-derived primary CRC cells, siRNA strategy was utilized to transiently knockdown AMPK $\alpha 1$ in primary $C R C$ cells. The two non-overlapping AMPK $\alpha 1$ siRNAs [32] both inhibited AMPK $\alpha 1$ expression and activation by ODE in primary cells (Figure 4D). As a consequence, ODE-exerted anti-proliferative
(Figure 4E) and pro-apoptotic (Figure 4F) activities were attenuated in AMPK $\alpha 1$-silenced primary cancer cells. Similar results were also observed in two other primary cancer cell lines (Data not shown). Together, these results suggest that AMPK activation mediates ODE-induced anti-CRC cell activity.

\section{ODE activates p53 signaling in CRC cells}

AMPK could activate p53-dependent apoptosis pathway in various cancer cells $[15,17,29,38,39]$. We showed that AMPK activation was required for vincristineinduced p53 activation and following melanoma cell apoptosis [14]. C6 ceramide and vincristine synergistically activated AMPK-p53 signaling to inhibit proliferation of multiple cancer cell lines [29]. Western blot results in Figure 5A showed that cytotoxic ODE $(25-200 \mu \mathrm{g} /$ $\mathrm{mL}$ ) treatment in HCT-116 cells induced significant p53 activation, which was tested by p53 phosphorylation (at Ser-15) and upregulation (Figure 5A). Significantly, the co-immunoprecipitation (Co-IP) assay results in Figure 5B
A.

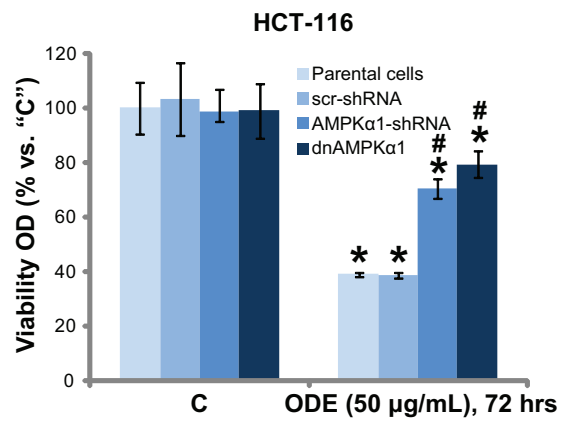

D.

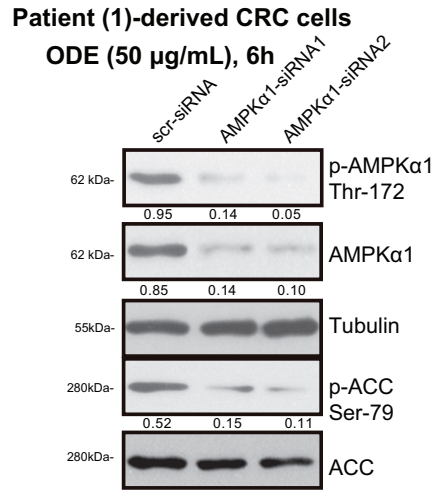

B.

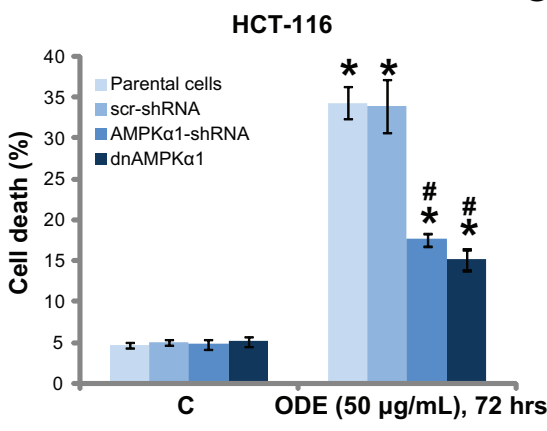

E. Patient (1)-derived CRC cells

F.

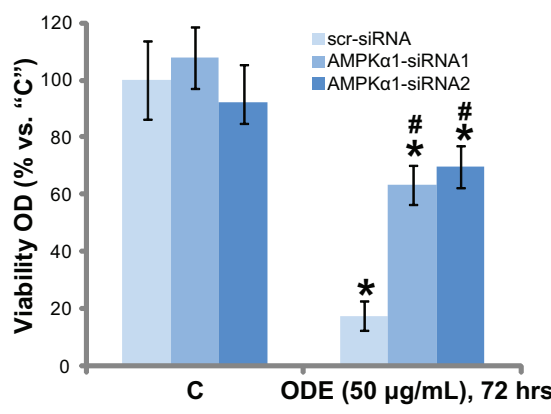

C.
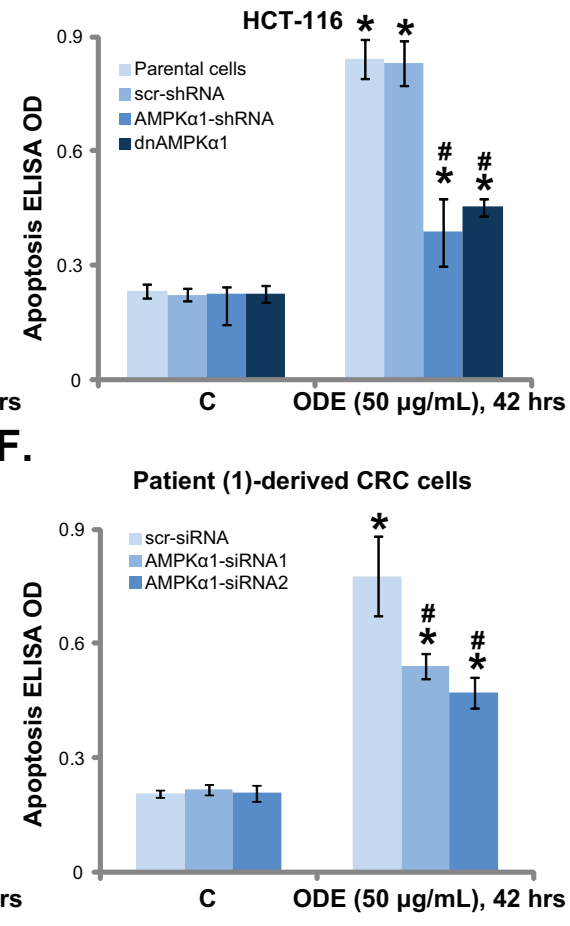

Figure 4: AMPK activation is required for ODE-induced anti-CRC cell activity. Stable HCT-116 cells expressing scramble control shRNA ("scr-shRNA"), AMPK $\alpha 1$-shRNA or dominant negative (dn)-AMPK $\alpha 1$ ("dnAMPK $\alpha 1$ ") as well as their parental cells were treated with or without applied ODE, cells were further cultured, cell viability (A., MTT assay), cell death (B., trypan blue staining assay) and cell apoptosis (C., Histone DNA ELISA assay) were tested. Primary CRC cells (patient-1-dervied), transfected with scramble control siRNA ("scr-siRNA") or AMPK $\alpha 1$-siRNA (-1/-2), were treated with ODE for indicated time, expressions of listed proteins were shown D., cell viability E. and apoptosis F. were tested similarly. Kinase phosphorylations were quantified (D). Data in this figure were repeated three times, and similar results were obtained. * $p<0.05$ vs. "C" of "scr-shRNA"/ "scr-siRNA" group. \# $p<0.05$ vs. "ODE" of "scr-shRNA"/ "scr-siRNA" group. 
showed that ODE treatment induced AMPK $\alpha 1$ and p53 association in HCT-116 cells. More importantly, activated AMPK $\alpha 1$ (p-Thr-172) and activated p53 (p-Ser-15) formed a complex in ODE-treated HCT-116 cells (Figure 5B). The "INPUT" results in Figure 5C again confirmed AMPK and p53 activation by ODE in HCT-116 cells. The above results suggest a potential role of AMPK in ODE-induced p53 activation in CRC cells. To support this hypothesis, we once again utilized same genetic strategies. Remarkably, as shown in Figure 5C, AMPK $\alpha 1$ shRNA knockdown or dominant negative mutation dramatically inhibited ODE-induced p53 activation in HCT-116 cells, suggesting that AMPK might be important for $\mathrm{p} 53$ activation in ODE-treated cells.

To study the role of p53 in ODE-induced antiCRC cell activity, we once again applied shRNA strategy to selectively and stably knockdown p53 in HCT-116 cells (See method in our previous study [29]). Results showed that ODE-induced anti-proliferation (MTT viability reduction, Figure 5E) and apoptosis (Figure
5F) were largely attenuated in p53-sileced stable HCT116 cells. Note that we utilized two non-overlapping p53 shRNAs, each showed high efficiency in downregulating p53 expression (Figure 5F, upper panel) and inhibiting ODE's actions in HCT-116 cells (Figure 5E and 5F). In primary human CRC cells (Patient-1), ODE treatment also induced significant p53 activation (Ser-15 phosphorylation and upregulation) (Figure 5G). Such an effect was again inhibited by AMPK $\alpha 1$ siRNA (Figure $5 \mathrm{H}$ ). Similar results were also seen in two other patient-derived CRC cell lines (Data not shown). Together, these results show that ODE activates AMPK-dependent p53 signaling to inhibit CRC cells.

\section{ODE inhibits HCT-116 xenograft growth in SCID mice}

The in vivo anti-CRC activity by ODE was also tested. As described, HCT-116 cells were injected into the SCID nude mice to create mice xenografts. These

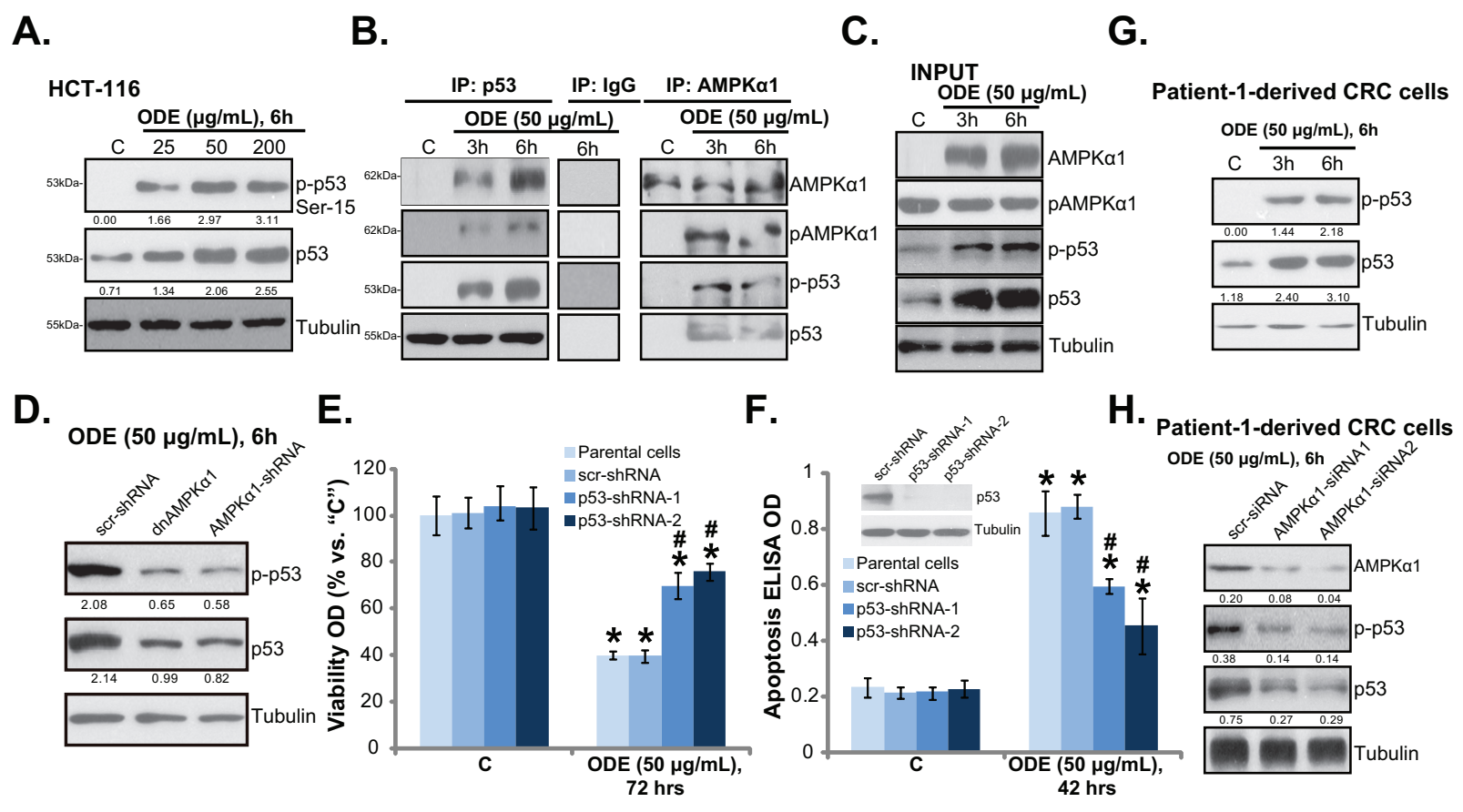

Figure 5: ODE activates p53 signaling in CRC cells. HCT-116 cells were treated with or without ODE at applied concentrations, cells were further cultured, expressions of listed proteins were tested by Western blots A and $\mathbf{C}$., the association between AMPK $\alpha 1$ (regular and p-) and p53 (regular and p-) was examined by co-immunoprecipitation (“Co-IP”) assay B., IgG was also included as a Co-IP control (B). Stable HCT-116 cells expressing scramble-shRNA ("scr-shRNA"), AMPK $\alpha 1$-shRNA or dominant negative (dn)-AMPK $\alpha 1$ ("dnAMPK $\alpha 1$ ”) were treated with applied ODE, p53 (regular and p-) and Tubulin expressions were tested by Western blots D. Stable HCT-116 cells expressing scramble-shRNA ("scr-shRNA") or p53-shRNA ("-1/-2") as well as their parental cells were treated with applied ODE, cell viability (MTT assay, E.) and cell apoptosis (Histone DNA ELISA assay, F.) were tested, expression of p53 in these cells was also shown (F, upper panel). p53 (regular and p-) and Tubulin expressions in ODE $(50 \mu \mathrm{g} / \mathrm{mL}$ )-treated primary CRC cells (patient-1 derived) were shown G. p53 (regular and p-) and AMPK $\alpha 1$ expressions in ODE $(50 \mu \mathrm{g} / \mathrm{mL})$-treated primary CRC cells with scramble control siRNA ("scr-siRNA") or AMPK $\alpha 1$ siRNA ("-1/-2") were shown H. Kinase phosphorylations and p53 expression were quantified. Data in this figure were repeated three times, and similar results were obtained. * $p<0.05$ vs. "C" of "scr-shRNA" group. " $p<0.05$ vs. "ODE" of "scr-shRNA" group. 
mice were subjected to ODE administration. Tumor growth curve results in Figure 6A showed that ODE administration significantly inhibited HCT-116 xenograft growth in SCID mice. The in vivo anti-HCT-116 activity of ODE was again dose-dependent, the high-dose ODE ("HD ODE", $1.0 \mathrm{~g} / \mathrm{kg}$, i.p., daily) was more potent than low-dose ODE ("LD ODE", $0.2 \mathrm{~g} / \mathrm{kg}$, i.p., daily) in suppressing HCT-116 xenografts (Figure 6A). Further, tumor daily growth was also significantly inhibited in ODE-treated mice (Figure 6B). Once again "HD ODE" group showed slower tumor daily growth than the "LD ODE" group (Figure 6B). In addition, the tumor weights at week-6 of ODE-treated groups were lower than that of vehicle (Saline) group (Figure 6C). These results suggest that ODE administration inhibited HCT-116 xenograft growth in SCID mice. We failed to detect any obvious deleterious effects in experimental mice. Mice body weights were not affected by the ODE regimens (Figure 6D), indicating the relative safety of the ODE treatments. Together, we show that ODE i.p. administration potently inhibits HCT116 xenograft growth in SCID mice. Next, xenografted tumors were isolated. Western blot analyzing these tumor tissues demonstrated significant AMPK activation (AMPK $\alpha 1 /$ ACC phosphorylations), p53 activation (Ser15 phosphorylation and upregulation) and mTORC1 in- activation (p-S6K1 inhibition) by ODE administration in vivo (Figure 6E). The in vivo activity of ODE on these signalings was again dose-dependent (see Figure 6E, quantification), and was observed in tumor tissues 3 days and 6 days after initial ODE administration (Figure 6E). Therefore, in line with in vitro findings, these results suggest that ODE administration results in AMPK-p53 activation and mTORC1 inhibition in xenografted HCT116 tumors.

\section{DISCUSSIONS AND CONCLUSIONS}

mTOR signaling is an important target for CRC intervention [40]. Our previous study has shown that INK128, a novel mTOR kinase inhibitor, induced significant anti-tumor activity in preclinical CRC models [2]. AMPK is known to inhibit mTORC1 through at least two distinct mechanisms. First, AMPK phosphorylates and activates TSC2 (Tuberous sclerosis protein 2), a negative regulator of mTOR, to inactivate mTORC1. Second, AMPK direct phosphorylates and in-activates of Raptor (regulatory associated protein of mTOR), which is a key functional component of mTORC1 [41]. Here, we showed that ODE activated AMPK signaling to inhibit mTORC1

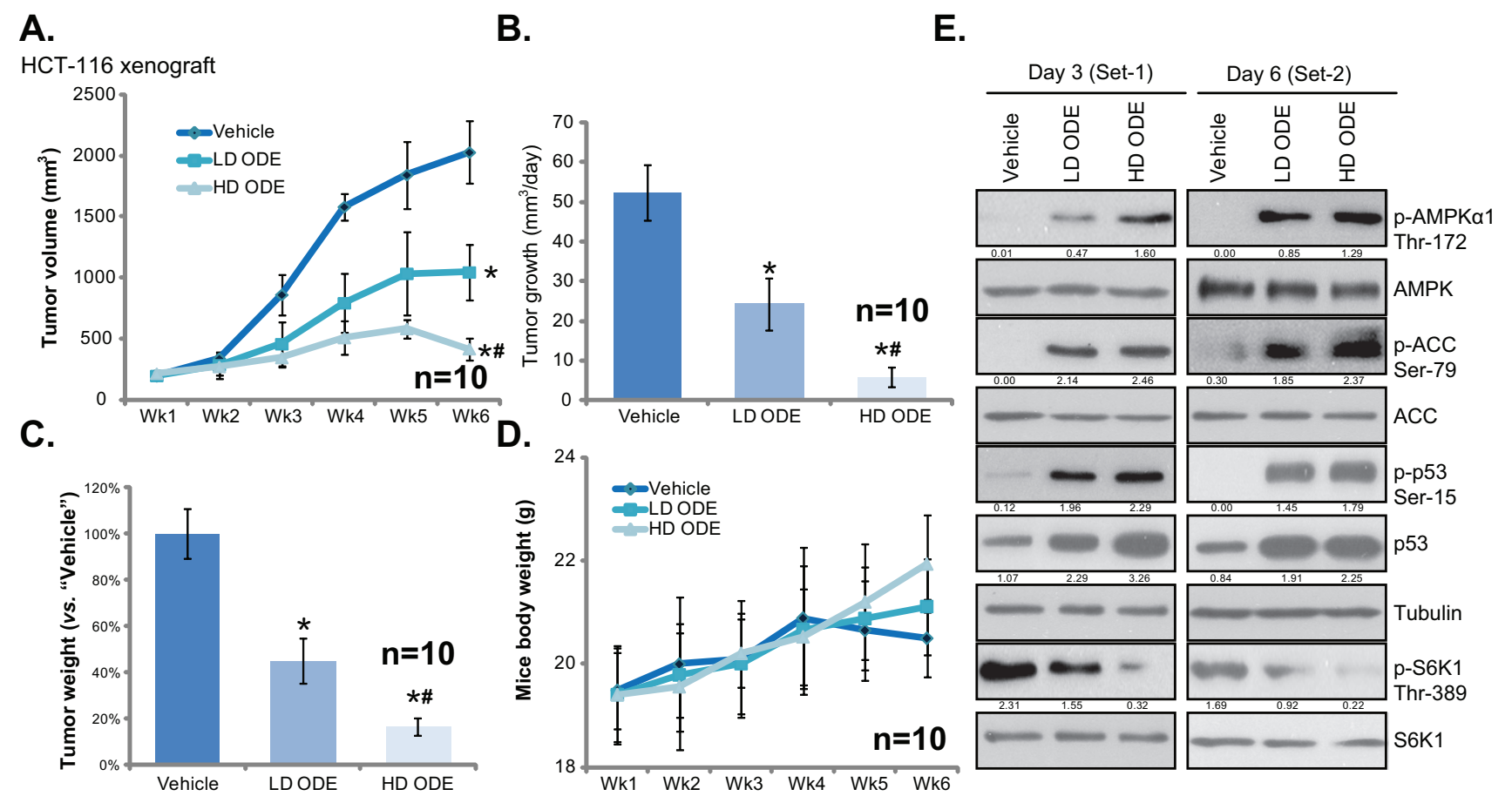

Figure 6: ODE inhibits HCT-116 xenograft growth in SCID mice. Weekly HCT-116 xenograft tumor growth curve A. and mice body weight curve D. with indicated treatment: Saline ("Vehicle"), low-dose of ODE ( $0.2 \mathrm{~g} / \mathrm{kg}$, i.p., daily, "LD ODE"), high-dose of ODE $(1.0 \mathrm{~g} / \mathrm{kg}$, i.p., daily, "HD ODE"), were shown (A). Tumor daily growth B. and tumor weights at week-6 C. were also presented. Three and six days after initial ODE administration, one mice per group were sacrificed, tumor tissues were removed and were subjected to Western blot assay of listed proteins E. Kinase phosphorylations and p53 expression were quantified (E). In vivo experiments were repeated twice, and similar results were obtained * $p<0.05$ vs. "Vehicle" group. " $p<0.05$ vs. "LD ODE" group. 
in CRC cells. S6K1 phosphorylation, the indicator of mTORC1 activation, as well expression of mTORC-1regulated genes (Bcl-2 and HIF-1 $\alpha$ ) were all inhibited in ODE-treated CRC cells. Reversely, inhibition or silence of AMPK restored mTORC1 activation and Bcl-2/HIF$1 \alpha$ expression. These results suggest that ODE activates AMPK signaling to inhibit cancer-promoting mTORC1 signaling in CRC cells.

The role of p53 in cell apoptosis has been wellestablished. Recent studies have shown that ODE-induced anti-cancer activity could be through activating p53 signaling [10]. Thus, one important finding of this study is that AMPK is also important for p53 activation by ODE in CRC cells. Our results showed that activated AMPK $\alpha 1$ formed a complex with p53 in ODE-treated CRC cells, which might be important for subsequent p53 activation and CRC cell apoptosis. Blockage of this complexation, though shRNA-mediated knockdown of p53 or AMPK $\alpha 1$, or by AMPK $\alpha 1$ mutilation, inhibited ODE-induced p53 activation and subsequent $\mathrm{CRC}$ apoptosis.

It should be noted that inhibition of AMPK via the methods described did not result in total abolition of ODEmediated cytotoxic effects against CRC cells. Meanwhile, p53 shRNA stable knockdown only inhibited (but not reversed) ODE's actions. Therefore, it is likely that other signalings, proposed by a number of other studies [9-13], could also contribute to the actions by ODE in CRC cells. Thus, it will be interesting to understand the relationship between AMPK signaling and these other possible pathways in mediating ODE's effects. Further studies will also be needed to explore the potential upstream kinases for AMPK activation by ODE.

The other important funding of the study is that i.p. injection of ODE at well-tolerated doses significantly inhibited HCT-116 xenograft growth in nude mice. Further, AMPK activation, mTORC1 inhibition and p53 activation were also observed in ODE-treated HCT-116 tumors. These results suggest that ODE could be further investigated as a novel and promising anti-CRC agent.

\section{MATERIALS AND METHODS}

\section{Ethics}

All methods listed in the study were carried out in accordance with the approved guidelines by authors' institutions (Nanjing University of Chinese Medicine, Nanjing Medical University and Jiangsu University).

\section{Chemicals, reagents and antibodies}

Oldenlandia diffusa extracts (ODE) were purified and provided by Nanjing University Of Chinese Medicine (Nanjing, China). The caspase-3 specific inhibitor AcDEVD-CHO, the caspase-9 inhibitor Ac-LEHD-CHO and the pan caspase inhibitor Ac-VAD-CHO were purchased from Calbiochem (La Jolla, CA). B-cell lymphoma 2 (Bcl-2) antibody (sc-7382), p53 antibody (sc-162), and hypoxia-inducible factor $1-\alpha$ (HIF-1 $\alpha$ ) antibody (sc10790) were obtained from Santa Cruz Biotechnology (Santa Cruz, CA). Antibodies of p-AMPKal (Thr172, \#2531), AMPK $\alpha$ (\#2532), acetyl-CoA Carboxylase (ACC, \#3662), p-ACC (Ser79, \#3661), p-p53 (Ser15, \#9284), p70 S6 Kinase (S6K1 \#9202), p-S6K1 (Thr389, \#9205), cleaved PARP (\#5625), cleaved-caspase-3 (\#9664) and $\beta$-Tubulin (\#2146) were purchased from were obtained from Cell Signaling Technology (Beverly, MA).

\section{Cell culture}

Human CRC cell lines, including HCT-116, DLD1, HT-29 and Lovo were cultured as described [2]. For all the cell lines, DNA fingerprinting and profiling were performed every 6 months to confirm the origin of the cell line, and to distinguish the cell line from cross-contamination. All cell lines were subjected to mycoplasma and microbial contamination examination. Population doubling time, colony forming efficiency, and morphology under phase contrast were also measured every 6 months under defined conditions to confirm the phonotype of cell line.

\section{Primary colon cancer cell isolation and culture}

Three patients with primary colon cancer administrated at Wuxi People's Hospital of Nanjing Medical University were enrolled in the study. Patient-1, male, 62 years old, Grade I, T2; Patient-2, male, 60 years old, Grade II, T3; Patient-3, male, 56 years old, Grade II, T2; As previously described [2], the surgery-isolated colon cancer specimens were thoroughly washed and minced into small pieces. Samples were then mechanically dissociated and filtered via a $70 \mu \mathrm{m}$ strainer. Single-cell suspensions of the colon cancer cells were achieved by re-suspending cells in $0.15 \%(\mathrm{w} / \mathrm{v})$ collagenase I (Sigma) dissolved in DMEM for $1 \mathrm{~h}$, individual cells were pelleted and rinsed twice with DMEM before re-suspending in the cell culture medium as described [2]. The study was approved by the institutional review board (IRB) of all authors' institutions. All clinical investigations were conducted according to the principles expressed in the Declaration of Helsinki. The protocol was approved by authors' institutions. Written-informed consent was obtained from all subjects.

\section{Methyl thiazol tetrazolium (MTT) assay of cell proliferation}

Cell proliferation was assessed via the MTT (Sigma) assay as described [2, 3, 27, 28]. 


\section{BrdU incorporation assay of cell proliferation}

The proliferation of CRC cells was also estimated via the incorporation of 5-bromo-2'-deoxyuridine (BrdU). Briefly, cells $\left(0.8 \times 10^{4} /\right.$ well $)$ were exposed to applied ODE treatment. Afterwards, BrdU $(10 \mu \mathrm{M}$, Roche Diagnostics, Shanghai, China) was added to the medium, and then the cells were incubated for another $16 \mathrm{~h}$. Next, the cells were fixed, and BrdU incorporation was determined with a cell proliferation enzyme-linked immunosorbent assay (ELISA) kit (Roche Diagnostics) according to the manufacturer's instructions. ELISA OD was utilized as a quantitative measurement of cancer cell proliferation.

\section{Colonies formation assay}

After applied ODE treatment, CRC cells were suspended in $1 \mathrm{~mL}$ of DMEM containing $0.25 \%$ agar (Sigma). The cell suspension was then added on the top of a pre-solidified $100 \mathrm{~mm}$ culture dish. After 10 days of incubation, the number of colonies were fixed, stained and manually counted.

\section{Trypan blue staining assay of cell death}

As described previously [2], following applied treatment, the cell death percentage was determined by counting cells via a hemocytometer, supplemented with trypan blue, which stains the cytoplasm of dead cells [29]. Cell death percentage $=$ the number of trypan blue stained cells/the number of total cells $(\times 100 \%)$.

\section{Assay of caspase-3 activity}

As described [30], to test caspase-3 activity, $20 \mu \mathrm{g}$ of cytosolic protein extracts per sample were mixed with the caspase assay buffer [30] and the caspase-3 substrate Ac-DEVD-AFC $(15 \mu \mathrm{g} / \mathrm{mL})$ (Calbiochem). After $1 \mathrm{~h}$ incubation at $37{ }^{\circ} \mathrm{C}$, the released $\mathrm{AFC}$ was measured through a Shimadzu FC 5300 spectrofluorometer with excitation at $400 \mathrm{~nm}$. The caspase- 3 activity of ODEtreatment group was normalized to that of untreated control group.

\section{Histone-DNA ELISA assay}

Cell apoptosis was quantified by Histone-DNA ELISA PLUS kit (Roche Applied Science, Shanghai, China) according to the manufacturer's protocol [3, 27, 28].

\section{Apoptosis assay by Annexin V fluorescence- activated cell sorting (FACS)}

The FACS detecting CRC cell apoptosis was described in our previous study [28]. Propidium iodide (PI) negative and Annexin V positive cells were gated as early apoptotic cells, and PI positive and Annexin V positive cells were gated as late apoptotic cells.

\section{TUNEL assay of apoptosis}

Cell apoptosis was also detected by the TUNEL (Terminal Deoxynucleotidyl Transferase dUTP Nick End Labeling) In Situ Cell Apoptosis Detection Kit (Roche, Shanghai, China), according to the manufacturer's instructions. TUNEL positive nuclei ratio was recorded.

\section{Western blots}

Western blots were performed as previously reported $[2,3,27,28]$. Blot intensity was quantified by ImageJ software (NIH) after normalization to corresponding loading control.

\section{Co-immunoprecipitation (Co-IP)}

As described [31], after applied treatment, 1000 $\mu \mathrm{g}$ of cell lysates per sample were pre-cleared with 30 $\mu \mathrm{L}$ of protein A/G PLUS-agarose (Santa Cruz) for $1 \mathrm{~h}$. Next, the lysates were centrifuged for $5 \mathrm{~min}$ at $4{ }^{\circ} \mathrm{C}$ in a micro-centrifuge to remove nonspecific aggregates. The supernatant was then rotated overnight with 0.1-0.25 $\mu \mathrm{g}$ of indicated primary antibody (anti-AMPK $\alpha 1 /$ anti-p53) (Santa Cruz). The protein A/G PLUS-agarose $(35 \mu \mathrm{L} /$ sample) was then added to the supernatants at $4{ }^{\circ} \mathrm{C}$ for 4 h. Pellets were washed six times with PBS, resuspended in lysis buffer, and then assayed by Western blots.

\section{Short hairpin RNA (shRNA) and stable cells selection}

As described in our previous studies [29], lentiviral particles containing the p53-shRNA-1 (Santa Cruz, sc29435-V), p53 shRNA-2 (Genechem, Shanghai, China) or AMPK $\alpha 1$ shRNA (Santa Cruz, sc-29673-V) (10 $\mu \mathrm{L} /$ well) were added to cultured CRC cells for $24 \mathrm{~h}$. Afterwards, cell culture medium was replaced with puromycin $(0.5$ $\mu \mathrm{g} / \mathrm{mL}$ )-containing complete medium. The medium was renewed every 2-3 days until resistant stable colonies were formed. Expression of targeted protein (AMPK $\alpha 1$ and p53) was always determined by Western-blots in resulting stable cells. Same amount of scramble control shRNA lentiviral particles (Santa Cruz, sc-108080) were added to control cells.

\section{AMPK dominant negative mutation}

The dominant-negative ( $\mathrm{dn}$ ) mutant of AMPK- $\alpha 1$ (AMPK- $\alpha 1-T 172 \mathrm{~A}$ ) construct was described previously [29]. dn-AMPK- $\alpha 1$ cDNA $(0.25 \mu \mathrm{g} / \mathrm{mL})$ was transfected to CRC cells via the Lipofectamine 2000 protocol [29], stable cells were selected through neomycin $(2 \mu \mathrm{g} / \mathrm{mL})$. 
Transfection efficiency was again determined by Western blots in resulting stable cells.

\section{AMPKa1 siRNA knockdown in primary human CRC cells}

The AMPK- $\alpha 1$ siRNA(-1) (5'-GCAUAUGCUGC AGGUAGAU-3'), the AMPK- $\alpha 1$ siRNA(-2) (5'-AAGG AAAGTGAAGGTGGGCAA-3') and the scramble control siRNA were all provided by Dr. Biao Xu's Lab [32]. Transient transfection was performed by the Lipofectamine 2000 reagent (Roche) according to the manufacturer's instructions. Transfection efficiency was determined by Western blots in resulting cells.

\section{Tumor xenografts}

The mice experiments were performed based on the protocol described in our previous studies $[4,29]$ with minor modifications. The male severe combined immuno-deficient (SCID) $n u / n u$ mice were implanted s.c. with HCT-116 cells $\left(5 \times 10^{6}\right.$ per mouse). When the tumors reached the average volume of $200 \mathrm{~mm}^{3}$, the mice were divided into three groups: Saline ("Vehicle"), low-dose of ODE group $(0.2 \mathrm{~g} / \mathrm{kg}$, i.p. $)$ and high-dose of ODE group $(1.0 \mathrm{~g} / \mathrm{kg}$, i.p.). ODE was freshly prepared and administered daily for a total of 30 days. Tumor volumes were recorded weekly, calculated via the following formula: $\pi / 6 \times$ larger diameter $\times(\text { smaller diameter })^{2}$. The mice were maintained as described [29]. All studies were performed in accordance with the standards of ethical treatment approved by the Institutional Animal Care and Use Committee (IACUC) and Association for the Assessment and Accreditation of Laboratory Animal Care (AAALAC). The protocol was approved by authors' institutions (Nanjing University of Chinese Medicine, Nanjing Medical University and Jiangsu University).

\section{Statistical analysis}

Each experiment was repeated a minimum of three times, with similar results obtained each time. Data were presented as mean \pm standard deviation (SD). Statistics were analyzed by one-way ANOVA followed by a Scheffe' and Tukey Test by SPSS 15.0 software (SPSS Inc., Chicago, IL). Significance was chosen as $p<0.05$. The concentrations of agents applied and the treatment durations were chosen based on published literatures and results from our pre-experiments. IC-50 was also calculated by SPSS software [2].

\section{ACKNOWLEDGMENTS}

This work was supported by the National Natural Science Foundation (No. 81273717, 81472305, 81472786), and the Six Talents Peak Project of Jiangsu
Province (2014-WSW-061, WSN-012). The funders had no role in study design, data collection and analysis, decision to publish, or preparation of the manuscript.

\section{CONFLICTS OF INTERESTS}

All listed authors state no conflict of interests.

\section{Author contributions}

PL, MC, JC and MhW participated in the design of the study. PL, MC, JC, WL, MxW and MhW performed all the experiments. PL, MC, JC, and MhW conceived of the study. PL, MC, JC, and MhW participated in its design and coordination and helped to draft the manuscript. All authors have read and approved the final manuscript.

\section{REFERENCES}

1. Liscovitch M, Lavie Y. Cancer multidrug resistance: a review of recent drug discovery research. IDrugs. 2002; 5:349-355.

2. Li C, Cui JF, Chen MB, Liu CY, Liu F, Zhang QD, Zou J, $\mathrm{Lu} \mathrm{PH}$. The preclinical evaluation of the dual mTORC1/2 inhibitor INK-128 as a potential anti-colorectal cancer agent. Cancer Biol Ther. 2015; 16:34-42.

3. Chen MB, Wei MX, Han JY, Wu XY, Li C, Wang J, Shen W, Lu PH. MicroRNA-451 regulates AMPK/mTORC1 signaling and fascin1 expression in HT-29 colorectal cancer. Cell Signal. 2014; 26:102-109.

4. Chen MB, Wu XY, Tao GQ, Liu CY, Chen J, Wang LQ, Lu $\mathrm{PH}$. Perifosine sensitizes curcumin-induced anti-colorectal cancer effects by targeting multiple signaling pathways both in vivo and in vitro. Int J Cancer. 2012; 131:2487-2498.

5. Schmoll HJ, Stein A. Colorectal cancer in 2013: Towards improved drugs, combinations and patient selection. Nat Rev Clin Oncol. 2014; 11:79-80.

6. Kuipers EJ, Rosch T, Bretthauer M. Colorectal cancer screening--optimizing current strategies and new directions. Nat Rev Clin Oncol. 2013; 10:130-142.

7. Brouquet A, Nordlinger B. Metastatic colorectal cancer outcome and fatty liver disease. Nat Rev Gastroenterol Hepatol. 2013; 10:266-267.

8. Palta M, Czito BG, Willett CG. Colorectal cancer: adjuvant chemotherapy for rectal cancer-an unresolved issue. Nat Rev Clin Oncol. 2014; 11:182-184.

9. Ovesna Z, Vachalkova A, Horvathova K, Tothova D. Pentacyclic triterpenoic acids: new chemoprotective compounds. Minireview. Neoplasma. 2004; 51:327-333.

10. Gu G, Barone I, Gelsomino L, Giordano C, Bonofiglio D, Statti G, Menichini F, Catalano S, Ando S. Oldenlandia diffusa extracts exert antiproliferative and apoptotic effects on human breast cancer cells through ERalpha/ 
Sp1-mediated p53 activation. J Cell Physiol. 2012; 227:3363-3372.

11. Willimott S, Barker J, Jones LA, Opara EI. Apoptotic effect of Oldenlandia diffusa on the leukaemic cell line HL60 and human lymphocytes. J Ethnopharmacol. 2007; 114:290-299.

12. Gupta S, Zhang D, Yi J, Shao J. Anticancer activities of Oldenlandia diffusa. J Herb Pharmacother. 2004; 4:21-33.

13. Sadava D, Ahn J, Zhan M, Pang ML, Ding J, Kane SE. Effects of four Chinese herbal extracts on drug-sensitive and multidrug-resistant small-cell lung carcinoma cells. Cancer Chemother Pharmacol. 2002; 49:261-266.

14. Chen MB, Shen WX, Yang Y, Wu XY, Gu JH, Lu PH. Activation of AMP-activated protein kinase is involved in vincristine-induced cell apoptosis in B16 melanoma cell. J Cell Physiol. 2011; 226:1915-1925.

15. Chen MB, Wu XY, Gu JH, Guo QT, Shen WX, Lu PH. Activation of AMP-activated protein kinase contributes to doxorubicin-induced cell death and apoptosis in cultured myocardial H9c2 cells. Cell Biochem Biophys. 2011; 60:311-322.

16. Vakana E, Altman JK, Platanias LC. Targeting AMPK in the treatment of malignancies. J Cell Biochem. 2012; 113:404-409.

17. Cao C, Lu S, Kivlin R, Wallin B, Card E, Bagdasarian A, Tamakloe T, Chu WM, Guan KL, Wan Y. AMP-activated protein kinase contributes to UV- and $\mathrm{H} 2 \mathrm{O} 2$-induced apoptosis in human skin keratinocytes. J Biol Chem. 2008; 283:28897-28908.

18. Kang MR, Park SK, Lee CW, Cho IJ, Jo YN, Yang JW, Kim JA, Yun J, Lee KH, Kwon HJ, Kim BW, Lee K, Kang JS, Kim HM. Widdrol induces apoptosis via activation of AMP-activated protein kinase in colon cancer cells. Oncol Rep. 2012; 27:1407-1412.

19. Kim YM, Hwang JT, Kwak DW, Lee YK, Park OJ. Involvement of AMPK signaling cascade in capsaicininduced apoptosis of HT-29 colon cancer cells. Ann N Y Acad Sci. 2007; 1095:496-503.

20. Sauer H, Engel S, Milosevic N, Sharifpanah F, Wartenberg M. Activation of AMP-kinase by AICAR induces apoptosis of DU-145 prostate cancer cells through generation of reactive oxygen species and activation of c-Jun N-terminal kinase. Int J Oncol. 2012; 40:501-508.

21. Shao JJ, Zhang AP, Qin W, Zheng L, Zhu YF, Chen X. AMP-activated protein kinase (AMPK) activation is involved in chrysin-induced growth inhibition and apoptosis in cultured A549 lung cancer cells. Biochem Biophys Res Commun. 2012; 423:448-453.

22. Sun H, Yu T, Li J. Co-administration of perifosine with paclitaxel synergistically induces apoptosis in ovarian cancer cells: more than just AKT inhibition. Cancer Lett. 2011; 310:118-128.

23. Wang B, Wang XB, Chen LY, Huang L, Dong RZ. Belinostat-induced apoptosis and growth inhibition in pancreatic cancer cells involve activation of TAK1-AMPK signaling axis. Biochem Biophys Res Commun. 2013; 437:1-6.

24. Yang L, Zheng LY, Tian Y, Zhang ZQ, Dong WL, Wang $\mathrm{XF}$, Zhang XY, Cao C. C6 ceramide dramatically enhances docetaxel-induced growth inhibition and apoptosis in cultured breast cancer cells: a mechanism study. Exp Cell Res. 2015; 332:47-59.

25. Zhang WB, Wang Z, Shu F, Jin YH, Liu HY, Wang QJ, Yang Y. Activation of AMP-activated protein kinase by temozolomide contributes to apoptosis in glioblastoma cells via p53 activation and mTORC1 inhibition. J Biol Chem. 2010; 285:40461-40471.

26. Zheng QY, Jin FS, Yao C, Zhang T, Zhang GH, Ai X. Ursolic acid-induced AMP-activated protein kinase (AMPK) activation contributes to growth inhibition and apoptosis in human bladder cancer T24 cells. Biochem Biophys Res Commun. 2012; 419:741-747.

27. Chen MB, Shen WX, Yang Y, Wu XY, Gu JH, Lu PH. Activation of AMP-activated protein kinase is involved in vincristine-induced cell apoptosis in B16 melanoma cell. J Cell Physiol. 2010; 226:1915-1925.

28. Chen MB, Zhang Y, Wei MX, Shen W, Wu XY, Yao C, Lu $\mathrm{PH}$. Activation of AMP-activated protein kinase (AMPK) mediates plumbagin-induced apoptosis and growth inhibition in cultured human colon cancer cells. Cell Signal. 2013; 25:1993-2002.

29. Chen MB, Jiang Q, Liu YY, Zhang Y, He BS, Wei MX, Lu JW, Ji Y, Lu PH. C6 ceramide dramatically increases vincristine sensitivity both in vivo and in vitro, involving AMP-activated protein kinase-p53 signaling. Carcinogenesis. 2015; 36:1061-1070.

30. Hishita T, Tada-Oikawa S, Tohyama K, Miura Y, Nishihara T, Tohyama Y, Yoshida Y, Uchiyama T, Kawanishi S. Caspase-3 activation by lysosomal enzymes in cytochrome c-independent apoptosis in myelodysplastic syndromederived cell line P39. Cancer Res. 2001; 61:2878-2884.

31. Yu T, Ji J, Guo YL. MST1 activation by curcumin mediates JNK activation, Foxo3a nuclear translocation and apoptosis in melanoma cells. Biochem Biophys Res Commun. 2013; 441:53-58.

32. Zheng K, Lu H, Sheng Z, Li Y, Xu B. Low-concentration of perifosine surprisingly protects cardiomyocytes from oxygen glucose deprivation. Biochem Biophys Res Commun. 2016; 469:753-760.

33. Dong Y, Zhao J, Wu CW, Zhang L, Liu X, Kang W, Leung WW, Zhang N, Chan FK, Sung JJ, Ng SS, Yu J. Tumor suppressor functions of miR-133a in colorectal cancer. Mol Cancer Res. 2013; 11:1051-1060.

34. Schonewolf CA, Mehta M, Schiff D, Wu H, Haffty BG, Karantza V, Jabbour SK. Autophagy inhibition by chloroquine sensitizes HT-29 colorectal cancer cells to concurrent chemoradiation. World J Gastrointest Oncol. 2014; 6:74-82. 
35. Shi L, Jackstadt $\mathrm{R}$, Siemens $\mathrm{H}, \mathrm{Li} \mathrm{H}$, Kirchner $\mathrm{T}$, Hermeking H. p53-induced miR-15a/16-1 and AP4 form a double-negative feedback loop to regulate epithelialmesenchymal transition and metastasis in colorectal cancer. Cancer Res. 2014; 74:532-542.

36. Zheng B, Mao JH, Qian L, Zhu H, Gu DH, Pan XD, Yi F, Ji DM. Pre-clinical evaluation of AZD-2014, a novel mTORC1/2 dual inhibitor, against renal cell carcinoma. Cancer Lett. 2015; 357:468-475.

37. Toschi A, Lee E, Gadir N, Ohh M, Foster DA. Differential dependence of hypoxia-inducible factors 1 alpha and 2 alpha on mTORC1 and mTORC2. J Biol Chem. 2008; 283:34495-34499.

38. Jones RG, Plas DR, Kubek S, Buzzai M, Mu J, Xu Y, Birnbaum MJ, Thompson CB. AMP-activated protein kinase induces a p53-dependent metabolic checkpoint. Mol Cell. 2005; 18:283-293.

39. Ji C, Yang YL, Yang Z, Tu Y, Cheng L, Chen B, Xia JP, Sun WL, Su ZL, He L, Bi ZG. Perifosine sensitizes UVBinduced apoptosis in skin cells: new implication of skin cancer prevention? Cell Signal. 2012; 24:1781-1789.

40. Guertin DA, Sabatini DM. Defining the role of mTOR in cancer. Cancer Cell. 2007; 12:9-22.

41. Brunn GJ, Williams J, Sabers C, Wiederrecht G, Lawrence JC, Jr., Abraham RT. Direct inhibition of the signaling functions of the mammalian target of rapamycin by the phosphoinositide 3-kinase inhibitors, wortmannin and LY294002. EMBO J. 1996; 15:5256-5267. 